\title{
The Addition of Asynchronous Chat-Based Coaching to a Digital Behavioral Health Tool Promotes Support and Personalization
}

\author{
myStrength, Inc, Denver, CO, United States \\ Corresponding Author: \\ Alissa Link, MPH \\ myStrength, Inc \\ 1875 Lawrence Street, Suite 550 \\ Denver, CO, \\ United States \\ Phone: 5938200 \\ Email: alink@mystrength.com
}

Alissa Link, MPH; Amy Lukowski, PsyD; Abigail Hirsch, PhD

\section{Abstract}

Background: Digital behavioral health $(\mathrm{BH})$ tools can help improve or maintain $\mathrm{BH}$ conditions such as depression, anxiety, substance abuse, stress, chronic pain or insomnia; the efficacy of digital self-care tools can be enhanced via addition of human touch-points.

Objective: The objective of this study was to evaluate the feasibility and acceptability of adding asynchronous chat-based coaching to the myStrength digital BH platform.

Methods: Participants who were at least 18 years old, fluent in English and readily able to access the internet were recruited via Craigslist advertisements to participate in a pilot intervention. Participants were asked to use the digital tool at least 2 times per week and to communicate via online chat with a coach at least 2 times per week. Participants completed online surveys at 2 weeks, 4 weeks and 6 weeks, and completed a 30 minute telephone interview between 5 weeks and 6 weeks.

Results: Of 226 who responded to the advertisement, 200 people were invited to participate, 134 engaged in coaching initially and 96 completed the final interview. Seventy-eight percent of participants were female, $62 \%$ were white, $23 \%$ were black, $8 \%$ were Hispanic and $6 \%$ were Asian. One-fifth were in treatment with a BH provider, one-fifth received BH care from a medical provider, and $22 \%$ were taking BH medications. In response to the question, "On a scale of 0-10, how helpful has your coach been over the past 2-weeks," the mean score and standard deviation were $7.5 \pm 2.4$ at 2 weeks, $8.2 \pm 1.9$ at 4 weeks and $8.6 \pm 1.9$ at 6 weeks. Satisfaction with coaching was $7.6 \pm 2.5$ (out of 10) at 2 weeks and $8.3 \pm 1.9$ at 4 weeks. Participants felt that coaching increased the value of the digital tool, with a mean rating of $8.4 \pm 1.9$ (out of 10). Three-quarters of participants felt that the encouragement coaches provided was the most helpful aspect, followed by guidance to specific resources (53\%), support (51\%), connection to another person (42\%), and assurance (40\%). These findings were corroborated in the qualitative data; participants emphasized the value of having personalized suggestions amidst a large breadth of content, an outside perspective, and greatly appreciated the "human contact in a digital world." The coach-patient relationship strengthened over time (2-weeks vs 6-weeks): $78 \%$ vs $93 \%$ felt that the coach discussed things that were important to the participant; $78 \%$ vs $90 \%$ felt that the coach liked and understood them; $85 \%$ vs $93 \%$ felt the interaction with their coach was helpful; $77 \%$ vs $88 \%$ had confidence in the working relationship with their coach (all $P<0.05$ ). Eighty-one percent of participants reported that they would prefer to work with the same coach over time whereas $19 \%$ would have preferred to talk with a coach in real time.

Conclusions: The addition of asynchronous coaching to a digital BH platform was well received, perceived as helpful, and promoted a more personalized experience with the digital $\mathrm{BH}$ tool that improved over time; future studies will evaluate impact on clinical outcomes and engagement. The added human element helped participants feel supported in their struggles with BH issues and has the potential to help increase engagement to myStrength's effective, evidence-based, well-being resources.

(iproc 2018;4(2):e11797) doi: $\underline{10.2196 / 11797}$

\section{KEYWORDS}

behavioral health; chat; coaching 
Edited by T Hale; this is a non-peer-reviewed article. Submitted 02.08.18; accepted 29.08.18; published 17.09.18.

Please cite as:

Link A, Lukowski A, Hirsch A

The Addition of Asynchronous Chat-Based Coaching to a Digital Behavioral Health Tool Promotes Support and Personalization iproc 2018;4(2):e11797

URL: http://www.iproc.org/2018/2/e11797/

doi: $\underline{10.2196 / 11797}$

PMID:

(C)Alissa Link, Amy Lukowski, Abigail Hirsch. Originally published in Iproceedings (http://www.iproc.org), 17.09.2018. This is an open-access article distributed under the terms of the Creative Commons Attribution License (https://creativecommons.org/licenses/by/4.0/), which permits unrestricted use, distribution, and reproduction in any medium, provided the original work, first published in Iproceedings, is properly cited. The complete bibliographic information, a link to the original publication on http://www.iproc.org/, as well as this copyright and license information must be included. 